\title{
PROTECCIÓN DE DATOS Y PRIVACIDAD. ESTUDIO COMPARADO DEL CONCEPTO Y SU DESARROLLO ENTRE LA UNIÓN EUROPEA Y ESTADOS UNIDOS
}

\section{DATA PROTECTION AND PRIVACY. COMPARATIVE STUDY OF THE CONCEPT AND ITS DEVELOPMENT BETWEEN THE EUROPEAN UNION AND THE UNITED STATES}

\section{ITZIAR SOBRINO GARCÍA}

Doctoranda del Programa Ordenación Jurídica del Mercado de la Universidad de Vigo

El presente texto se encuentra cofinanciado con fondos del "Programa e axudas á etapa predoutoral" de la Xunta de Galicia (Consellería de Educación, Universidade e Formación Profesional)

Resumen: En el presente estudio se realiza un análisis del concepto legal de protección datos tanto a nivel normativo en la Unión Europa como en Estados Unidos, al igual que una breve visión de su evolución legislativa. El largo desarrollo teórico y normativo que ha habido en la Unión Europea deja entrever la importancia de la identidad digital de sus ciudadanos, mientras que la legislación norteamericana, más esparcida y sectorial, revela la fuerza que otorga al libre flujo de datos entre las entidades mercantiles. La importancia del presente estudio reside en que la comprensión de la concepción sobre la protección de datos en ambos sistemas, permitirá tener una mejor perspectiva sobre las debilidades y fortalezas que puedan existir en los acuerdos entre la Unión Europea y EE. UU.

Palabras clave: protección de datos, privacidad, intimidad. 
Abstract: In the present study, an analysis of data protection as a legal concept is carried out in the European Union and in the United States, as well as a brief overview of its legislative evolution. The theoretical and normative development that has taken place in the European Union reveals the importance of the digital identity of its citizens. Nevertheless, due to the characteristics of the American legislation, the free flow of data between entities has more strength. The importance of this study lies in the fact that the understanding of the data protection concept in both systems will allow us to have a better perspective on the weaknesses and strengths that may exist in the agreements for the transfer of personal data between the European Union and the USA.

Keywords: data protection, privacy, intimacy.

Recepción original: 06/02/2019

Aceptación original: 07/11/2019

Sumario: I. Introducción. II. Perspectiva sobre el origen del derecho a la intimidad y el desarrollo de la protección de datos en la Unión Europea. II. A. La vida privada y su configuración jurisprudencial en la Unión Europea. II. B. La configuración normativa del derecho a la protección de datos. II. B. 1. Convenio $\mathrm{n}^{\mathrm{o}} 108$ del Consejo de Europa para la protección de las personas con respecto al tratamiento automatizado de datos de carácter personal. II. B. 2. Directiva 95/46/CE, relativa a la protección de personas físicas en lo referido al tratamiento de datos personales y a su libre circulación. II. B. 3. El Reglamento (CE) $\mathrm{n}^{\circ} 45 / 2001$ del Parlamento Europeo y del Consejo. II. B. 4. La Directiva 2002/58/CE del Parlamento Europeo y del Consejo, de 12 de julio de 2002. II. B. 5. Directiva 2006/24/CE del Parlamento Europeo y del Consejo, de 15 de marzo de 2006. II. B. 6. El Reglamento (UE) no 2016/679 del Parlamento Europeo y del Consejo de 27 de abril de 2016. III. La visión norteamericana del concepto privacy y la protección de datos. III. A. El reconocimiento de la privacidad. III. B. La tendencia legislativa norteamericana sobre la privacidad. IV. Conclusiones.

\section{INTRODUCCIÓN}

En el mundo actual predominado por las Tecnologías de la Información y las Comunicaciones, la creación de productos y servicios cada vez más accesibles a los ciudadanos que impulsan nuevas formas de relacionarse y comunicarse, están a la orden del día. Es- 
tos avances han dado lugar a medios que permiten la gestión automatizada de la información, generando como consecuencia que el análisis y recogida de cantidades ingentes de datos sea posible. Esa accesibilidad y capacidad de procesamiento han traído consigo la necesidad de otorgar protección jurídica a los datos de carácter personal de las personas físicas, sobre todo a aquello que pueda afectar a la intimidad de las personas. En este sentido la Unión Europea ha regulado este tipo de relaciones buscando la aplicación coherente en el contexto de sus políticas.

No obstante, con la aparición de la Sociedad de la Información el acceso a todo tipo de bienes y servicios conlleva generalmente la entrega de datos personales, no siendo siempre conscientes los ciudadanos de ello. Esta situación se complica cuando dichos datos son recogidos por empresas situadas en países que no forman parte de la Unión Europea y que por lo tanto no ofrecen el mismo nivel normativo de protección. Por ello, se hizo necesaria la aparición de mecanismos que permitiesen el movimiento internacional de datos con terceros países: las transferencias internacionales de datos. Este mecanismo o elemento se ha puesto en marcha con diversos países siendo uno de los más polémicos el norteamericano por el sistema normativa y las políticas existentes en torno a la privacidad.

Este estudio se presenta entonces como un punto de partida para la comprensión de los elementos diferenciadores entre la cultura normativa europea y la estadounidense sobre la protección de datos y la privacidad. Para ello se analizará conceptualización jurídica de los datos personales en el ámbito de la UE, partiendo del derecho a la intimidad, así como la revisión normativa. Mientras que en el caso de EE. UU se llevará a cabo una revisión de la construcción jurisprudencial y aplicación normativa.

\section{PERSPECTIVA SOBRE EL ORIGEN DEL DERECHO A LA LA INTIMIDAD Y EL DESARROLLO DE LA PROTECCIÓN DE DATOS EN LA UNIÓN EUROPA}

La intimidad, el espacio personal, y la vida privada se presenta como el límite entre lo público y lo privado, entre aquello que resulta confidencial y lo que puede mostrarse. Ya en el campo sociológico se ha determinado que la invasión del espacio personal presenta una naturaleza aversiva ${ }^{1}$, y hay una necesidad de carácter univer-

${ }^{1}$ Vid. AIELLO, J., y T, AIELLO, T., "The development of personal space: proxemics behavior of children six through sixteen”, Human ecology, 2, 1974, págs.127-136. 
sal sobre la necesidad de preservar la intimidad ${ }^{2}$. La misma se ha encontrado vinculada a otros procesos típicos del comportamiento humano tales como la comunicación, la sensación de control o autonomía, el sentido de la identidad y la liberación emocional ${ }^{3}$. De esta forma la intimidad en el derecho se presenta como una manifestación jurídica de una necesidad inminentemente social, de esta forma se reconoce un ámbito inaccesible y reservado de cada individuo frente a terceros ${ }^{4}$. Las raíces de este derecho se encuentran en el reconocimiento de las personas como recipientes de información sobre ellas mismas, implicando el desarrollo de su personalidad la información que deciden comunicar a los demás. Donde ciertas características como la religión, política, opiniones u orientaciones sexuales, son más difíciles de compartir entre los individuos, requiriendo cierta protección ante posibles injerencias ${ }^{5}$.

Si bien, el fundamento y significado en el ámbito jurídico se encuentra en el principio de la dignidad de la persona y de la tutela de la persona individual. Garantizando la dignidad de la persona el pleno desarrollo de la personalidad individual, implicando, por una parte, el reconocimiento de la autodisposición, sin injerencias de terceros y, por otra parte, conllevando la autodeterminación que nace de la proyección humana, vinculada a la idea de intimidad perso$\mathrm{nal}^{6}$. Puede decirse entonces que el derecho a la intimidad se postula como un derecho de la personalidad, ya que constituye un bien instrumental para garantizar la libertad del individuo en el desarrollo de su propia vida. Por lo que la libertad individual se presenta como fundamento necesario de la dignidad humana, y el derecho a la intimidad se configura como elemento esencial para el desarrollo de la personalidad ${ }^{7}$.

Sin embargo, no puede hablarse de una configuración del derecho a la intimidad hasta finales del siglo XIX, existiendo con ante-

${ }^{2}$ Vid PERRY, A., RUBINSTEN, O., PELED, L., y SHAMAY-TSOORY, S., "Don't stand so close to me: A behavioral and ERP study of preferred interpersonal distance”, Neuroimage, 83, 2013, págs-761-769.

${ }^{3}$ Vid LÓPEZ CHAO, V.A., El impacto del diseño del espacio y otras variables socio-físicas en el proceso de enseñanza-aprendizaje, Tesis doctoral Universidad de la Coruña, 2016.

${ }^{4}$ Vid RUÍZ MIGUEL, C., La configuración constitucional del derecho a la intimidad, Tecnos, Madrid, 1995.

${ }^{5}$ Vid SAVIRIMUTHU, J., Security and Privacy. Volume III, Routledge, Oxford, 2016.

${ }^{6}$ Vid PÉREZ LUÑO, A.E., Derechos humanos, Estado de Derecho y Constitución, Tecnos, Madrid, 1986.

${ }^{7}$ Vid HERRÁN ORTIZ, A.I., El derecho a la protección de datos personales en la sociedad de la información, Universidad de Deusto, Bilbao, 2003. 
rioridad el reconocimiento de diferentes aspectos de la intimidad, pero sin haber una concepción global del derecho. Surge entonces, como preocupación por la protección de la esfera privada en reacción a las constantes intromisiones de la prensa en el ámbito personal y familiar. Este derecho se concibe como the right to be alone, el derecho a estar sólo, siendo reconocido inicialmente por los tribunales americanos y con posterioridad por la Declaración Universal de Derechos Humanos ${ }^{8}$. Se trataba de proteger el espacio íntimo, de la intromisión o injerencia de terceros, de decidir quién puede o no participar en las acciones o decisiones ocurridos en ese ámbito. En sí presenta la naturaleza de una libertad como ausencia de coacciones externas que dificulten las decisiones o acciones de una persona9. Estos primeros casos que llegaban ante los tribunales versaban sobre todo al derecho a la propia imagen, que se había considerado como un reflejo del derecho a la intimidad ${ }^{10}$.

Así pues, y debido a la trascendencia de la intimidad y la privacidad, en el ámbito europeo se recogió en el Convenio Europeo de Derechos Humanos, de 1950 concretamente en su artículo 8: “1. Toda persona tiene derecho al respeto de su vida privada y familiar, de su domicilio y de su correspondencia. 2. No podrá haber injerencia de la autoridad pública en el ejercicio de este derecho, sino en tanto en cuanto esta injerencia esté prevista por la ley y constituya una medida que, en una sociedad democrática, sea necesaria para la seguridad nacional, la seguridad pública, el bienestar económico del país, la defensa del orden y la prevención del delito, la protección de la salud o de la moral, o la protección de los derechos y las libertades de los demás." Presentándose como uno de los marcos base para la protección y el desarrollo de los derechos humanos y de las libertades fundamentales.

El término intimidad se ha utilizado también junto a privacidad, vida privada o ámbito íntimo, entre otros. Y aunque a primera vista pueda parecer que dicen lo mismo, con el paso del tiempo el signi-

${ }^{8} \mathrm{Vid}$. BRANDEIS, L.D., y WARREN, S.D., "The right of privacy (the implicit made explicit)", Harvard Law Review, 5, 1890, págs.193-220.

9 Vid. MARTÍNEZ DE PISÓN, J., "El derecho a la intimidad: de la configuración inicial a los últimos desarrollos en la jurisprudencia constitucional", Anuario de Filosofía del Derecho, 32, 2016, págs. 409-430.

10 Vid. MAESTRE RODRÍGUEZ, J.A., "La intimidad: El derecho de autodeterminación personal”, en C. VILLAGRASA ALCAIDE (coord.), Nuevas tecnologías de la información y derechos humanos, (págs. 275-284), Cedecs, Barcelona, 2003.

(C) UNED. Revista de Derecho UNED, núm. 25, 2019 
ficado de los diferentes términos se ha ido determinando y decantando finalmente por intimidad y privacidad ${ }^{11}$.

Los distintos términos que acuñan el ámbito de la vida privada (intimidad, secreto, confidencialidad, etc.) presentan una identidad significativa, y representan la idea de la existencia de una esfera privada, donde cada persona tiene potestad para decidir lo que le afecta y prohibir las intromisiones no deseadas.

\section{II.A. Las concepciones material y formal del derecho a la intimidad}

La concepción material de este derecho ha tenido un especial estudio en la doctrina y jurisprudencia alemanas, a través de la conocida teoría de las esferas. Con esta aproximación material se supone que cuanto más próximo se encuentre el dato difundido al núcleo básico de lo que se considera materialmente íntimo, mayor relevancia será la exigida a la información para que sea considerada constitucionalmente legítima. En atención a dicho planteamiento se produce la división en las siguientes ${ }^{12}$ :

1. Intimsphäre (la esfera íntima): se refiere a aquello más secreto del individuo, lo relacionado con sus opiniones, decisiones y acciones íntimas y más personales. Esa primera esfera correspondería al círculo más cercano del individuo.

2. Privatsphäre (la esfera privada): esta segunda esfera contempla un círculo más amplio donde la persona sigue ejerciendo su privacidad, su vida privada, su intimidad personal y familiar que quiere que esté asegurada frente a terceros.

3. Individualsphäre (la esfera individual): corresponde al último de los círculos de la intimidad antes de la vida pública, que se encontraría constituido por otros aspectos, como el honor o la imagen personal, que serían un reflejo de la personalidad del individuo.

Se puede apreciar que con la aproximación material a la noción de intimidad se ven cubiertas aquellas intromisiones que provienen de los medios de comunicación. Si bien, en el caso español, en la recepción de la noción material del derecho a la intimidad por la ju-

11 Vid. MARTÍNEZ DE PISÓN, J. "Vida privada e intimidad: implicaciones y perversiones", Anuario de Filosofía del Derecho, 14, 1997, págs. 717-738.

12 MARTÍNEZ DE PISÓN, J., "Vida privada..." op. cit., p. 717 y ss. 
risprudencia, se encuentra el referente de la STC 114/1984 que establece el criterio por el que resulta viable seleccionar diversos asuntos que en razón de su contenido pueden asociarse con la intimidad de la persona.

En cuanto a la concepción formal, en la misma prima la capacidad del titular del derecho para delimitar y decidir sobre la información que le concierna en su ámbito íntimo. Cuestión ya planteada por la doctrina norteamericana como se verá más adelante. No obstante, la concepción presentada por la teoría de las esferas constaba de un déficit por la posición secundaria que se le atribuía al afectado para delimitar el alcance de su ámbito intimo frente a terceros ${ }^{13}$.

\section{II.A. La vida privada y su configuración jurisprudencial en la Unión Europea}

El derecho a la intimidad se presenta entonces como la libertad para limitar o impedir el acceso al ámbito indisponible por los demás, para evitar o impedir injerencias externas hacia lo privado. Se presenta como un eslabón de los diversos que el ordenamiento ha generado para el libre desarrollo de la personalidad ${ }^{14}$. Y en este sentido la línea jurisprudencial lo ha entendido, concretamente el Tribunal Europeo de Derechos Humanos defiende un entendimiento amplio del derecho a la intimidad, presentándose como caso más paradigmático la sentencia de 16 de diciembre de 1992, el caso Nimietz c. Alemania. En ella el Tribunal consideró como restrictivo el reducir el ámbito al círculo interior en el que el individuo desarrolla su propia vida personal. Por ello, la vida privada debe englobar hasta cierto punto el derecho a establecer y desarrollar relaciones con otras personas. Dicha concepción amplia engloba actividades con relaciones relativas a la vida e identidad sexuales como con la sentencia del TEDH del 26 de marzo de 1985, Caso X e Y c. Países Bajos, la confidencialidad de datos sobre la salud, en el caso de la sentencia TEDH de 25 de febrero de 1997, Caso Z c. Finlandia, e incluso la elección del propio nombre, con la sentencia del TEDH de 22 de febrero de 1994, Caso Burghatz c. Suiza.

Se busca reconocer al individuo unos derechos que le corresponden por el hecho de ser persona. De tal forma que la persona no

13 Vid. MEDINA GUERRERO, M., La protección constitucional de la intimidad frente a los medios de comunicación, Tirant lo Blanch, Valencia, 2005.

14 Vid. CARRILLO, M., El derecho a no ser molestado. Información y vida privada, Aranzadi, Navarra, 2003. 
tiene que ejercitar acción alguna, ni cumplir otros requisitos que el mero hecho de serlo. En el ámbito europeo no se ha querido delimitar de manera exacta este derecho, ya que tiene un carácter expansivo que varía con el paso de los años. Por su parte el TJUE, ha abordado esta cuestión entendiendo que el derecho al respeto a la vida privada constituye un derecho fundamental protegido por el ordenamiento jurídico comunitario, encontrándose un ejemplo de ello en la STJUE de 5 de octubre de 1994, Caso X c. la Comisión de las Comunidades Europeas. En ella el TJUE estima el recurso de casación interpuesto por el demandante ante su sometimiento sin consentimiento, a una prueba de detección del SIDA de forma indirecta, lo que supuso la falta de aptitud física necesaria para ser contratado como mecanógrafo en la Comisión. Para dicha estimación, se basó en que el derecho al respeto a la vida y que se encontraba consagrado en el CEDH era uno de los derechos fundamentales y que, si el interesado se negaba a ser sometido a dicha prueba, tendría que haberse respetado su voluntad, no siendo posible su sometimiento a pruebas indirectas. El respeto de la vida privada exige a su vez que se respete la negativa del interesado en toda su extensión ${ }^{15}$.

En relación al alcance, el TEDH, en la sentencia de 24 de febrero de 1998, Caso Botta c. Italia, ha establecido límites, es decir, que debe ser entendido como derecho a la intimidad siempre que se muestre la existencia de un vínculo directo entre las medidas buscadas por el demandante y su vida privada y familiar. La protección que otorga el artículo 8.1 del Convenio Europeo de Derechos Humanos no se extiende a cualquier manifestación de la libre disposición individual sobre los aspectos de la vida de una persona, solamente con aquellas que tengan una relación expresa con la parte privada.

Por su parte, la STJUE de 20 de mayo de 2003, asuntos acumulados C-465/00, C-138/01 y C-139/01, Rechnungshof contra Österreichischer Rundfunk y otros y Christa Neukomm y Joseph Lauermann contra Österreichischer Rundfunk, ha afirmado que este derecho incluye también el no sufrir injerencias en la vida privada, que tiene que interpretarse en relación con el TEDH y su jurisprudencia, que ha aclarado que los términos de vida privada no pueden interpretarse restrictivamente ${ }^{16}$.

La privacidad es un concepto variable en atención a situaciones personales y sociales que hace referencia, no sólo a la intimidad,

15 Vid. TEJERINA RODRÍGUEZ, O., Seguridad del estado y privacidad, Reus, S.A, Madrid, 2014.

${ }_{16}$ En este sentido se encuentra Amann c. Suiza, no. 27798/1995, § 69, ECHR 2000-II. 
sino también al ámbito de la persona formado por su vida familiar, aficiones, bienes particulares y actividades personales.

Se puede determinar que la vida privada está configurada en función de dos ámbitos, uno interior, referido al individuo afectando sobre todo a su moralidad y a su pensamiento. Y, por otro lado, un ámbito externo en el que se le atribuyen a la persona las mismas facultades que sobre sí mismo, pero con referencia a los demás. Componiendo, por lo tanto, este ámbito los datos relativos a dicha persona, su domicilio, sus comunicaciones y sus relaciones personales y afectivas. En cualquiera de los casos la persona tiene derecho a controlarlos ${ }^{17}$.

\section{II.B. La configuración normativa del derecho a la protección de datos}

El desarrollo y el impacto de las nuevas tecnologías de la comunicación unido al derecho a la intimidad han dado lugar al reconocimiento del derecho a la protección de datos, así como una creciente sensibilización hacia el valor de los datos personales. Ya que, aunque las nuevas tecnologías y servicios beneficien tanto a las empresas como a los consumidores, también generan serios riesgos de privacidad Esta situación puede disminuir la confianza de las personas en empresas que recopilan datos para su producción de servicios ralentizando el desarrollo de la innovación, así como el uso y la adopción de nuevas tecnologías ${ }^{18}$.

Ocurre que en el caso de las nuevas tecnologías no se presentan solamente como una herramienta útil en las funciones de gestión y control económico en el ámbito público y empresarial, tienen también un interés especial en la unión con las telecomunicaciones y la apertura al mundo exterior. Si bien al no presentar unos límites claros se plantean una serie de dudas sobre el respeto de los derechos básicos de las personas y su estructura en la organización social ${ }^{19}$.

Por ello, el derecho a la protección de datos personales se presentó como la respuesta frente a un fenómeno inminente como era y es el desarrollo de la sociedad de la información existiendo posturas

17 Vid. REBOLlo DELGADO, L., (Vida privada y protección de datos en la Unión Europea, Dynkinson, S.L, Madrid, 2008.

${ }_{18} \mathrm{Vid}$. REDING, V., "The upcoming data protection reform for the European Union", International Data Privacy Law, 1, 2011, págs. 3-5.

19 Vid. DAVARA RODRÍGUEZ, M., Manual de Derecho Informático, Thomson Reuters, Pamplona, 2015.

(C) UNED. Revista de Derecho UNED, núm. 25, 2019 
enfrentadas sobre el reconocimiento de un nuevo derecho ${ }^{20}$ frente a la restructuración del derecho a la intimidad ${ }^{21}$. La protección se contempla como una evolución del derecho la vida privada surgida como consecuencia de la evolución tecnológica en el sector público y el privado. Haciendo evidente esta evolución la necesidad de protecciones adicionales para los individuos de los diferentes Estados ${ }^{22}$.

De esta forma se entiende que el derecho a la protección de datos en la UE conlleva el derecho del individuo a decidir cuándo y bajo qué circunstancias se pone a disposición su información personal, estando permitido el procesamiento de datos cuando la persona haya consentido o cuando así lo establezca la ley ${ }^{23}$, concepción y elementos que se han ido desarrollando a lo largo de la normativa europea.

II.B.1. Convenio $\mathrm{n}^{\circ} 108$ del Consejo de Europa para la protección de las personas con respecto al tratamiento automatizado de datos de carácter personal.

Así, el Convenio nº 108 , de 1981 , se estableció como el marco genérico de protección de la persona frente a las posibles lesiones del derecho a la intimidad. Todos los Estados miembros de la UE lo han ratificado, e incluso en 1999 se modificó para permitir que la propia UE fuera Parte del mismo. No obstante, este Convenio se encuentra además abierto a la adhesión de Estados no miembros, incluyendo países no europeos, teniendo un carácter universal. Así por ejemplo Uruguay, fue el primer país no europeo que se adhirió en agosto de 2013. Se convierte en el primer instrumento jurídicamente vinculante en el ámbito internacional adoptado en materia de protección de datos. En este Convenio se establecieron en las condiciones generales una serie de definiciones en su artículo segundo para aclarar y delimitar la afectación de la protección de datos. Además, este instrumento recoge una serie de principios sobre la protección de

${ }^{20}$ Vid. LUCAS MURILLO DE LA CUEVA, P., Informática y protección de datos personales. Estudios sobre la Ley Orgánica 5/92, de regulación del tratamiento automatizado de los datos de carácter personal, Centro de Estudios Constitucionales, Madrid, 1993.

${ }^{21}$ Vid. ORTÍ VALLEJO, A., Derecho a la intimidad e informática, Tutela de la persona por el uso de ficheros y tratamientos informáticos de datos personales. Particular atención a los ficheros de titularidad privada, Comares, Granada, 1994.

${ }^{22}$ Vid. KUNER, C., European data privacy law and online business, Oxford University Press, Nueva York, 2003.

${ }^{23}$ Vid. TINNEFELD, M.T., BUCHNER, B., PETRI, T., y HOF, H.J., Einführung in das datenschutzrecht. Datenschutz und informationsfreiheit in Europäischer sicht, De Gruyter Oldenbourg, Berlin, 2012. 
datos, señalando criterios para regular el flujo de datos, y estableciendo la creación de un Comité Consultivo, que estaría encargado de la formulación de propuestas para mejorar la aplicación del Convenio. El mismo se aplica a todo procesamiento de datos llevado a cabo tanto por el sector privado como público, protegiendo a las personas físicas contra los posibles abusos que puedan darse en la recogida y el tratamiento de datos personales, al mismo que tiempo que se buscaba regular los flujos transfronterizos de datos. Se incluyen una serie de principios que a día de hoy se mantienen en cierta medida en la legislación vigente.

Sin embargo, la eficacia del Convenio $\mathrm{n}^{\circ} 108$ fue decayendo y resultó incapaz de resolver los problemas en torno a la transmisión transfronteriza de datos, ya que, por una parte, solamente hubo un reducido número de nuevos países que firmaron el convenio. Y, por otro lado, no todos los países incluyeron el Convenio n ${ }^{\circ} 108$ en su derecho interno ${ }^{24}$. Por ello, la UE tomó la decisión de adoptar una directiva, escogiendo esa forma al ser regulación comunitaria vinculante para los países miembros y dando lugar a la Directiva 95/46/ $\mathrm{CE}$, relativa a la protección de personas físicas en lo referido al tratamiento de datos personales y a su libre circulación ${ }^{25}$.

II.B.2. Directiva 95/46/CE, relativa a la protección de personas físicas en lo referido al tratamiento de datos personales y a su libre circulación

De esta forma, es con la Directiva 95/46/CE de la protección de las personas frente al tratamiento de sus datos personales y de la libre circulación de esos datos, cuando se establece la protección de las libertades y los derechos fundamentales de las personas físicas y, en particular, del derecho a la intimidad, en lo que respecta al tratamiento de datos personales.

Tres objetivos clave fueron los que configuraron la Directiva 95/46/CE, el primero aproximar y armonizar las disposiciones de los Estados miembros sobre la protección de la intimidad de las personas frente a la informática, el segundo fue promover una normativa sobre redes públicas digitales y de comunicaciones, y finalmente,

${ }^{24}$ Vid. RUIZ CARRILLO, A., La protección de datos de carácter personal, Bosh, Barcelona 2001.

${ }^{25} \mathrm{Vid}$. HEREDERO HIGUERAS, M., La Directiva comunitaria de protección de datos de carácter personal, Aranzadi, Pamplona, 1997.

(C) UNED. Revista de Derecho UNED, núm. 25, 2019 
avanzar en la regulación para alcanzar un nivel adecuado en este sistema.

Reguló también uno de los conceptos clave en el ámbito de protección de datos: la transferencia internacional de datos. Es decir, cuando los datos personales tratados por un responsable o encargado de tratamiento en el Espacio Económico Europeo son enviados a un tercer país u organización internacional, situada fuera de dicho territorio. Se establecieron una serie de principios en la cesión de datos a terceros países, establecidos fuera de la UE, que implicaban fundamentalmente, que la cesión debía ser acorde al derecho nacional y que el país que recibiese esos datos tuviese un nivel de garantía adecuado, pudiendo realizarse únicamente en estas condiciones. Y que trajo consigo la Decisión 2000/520/CE de 26 de julio de la Comisión Europea, el Safe Harbor, donde se recogían los principios sobre protección de datos que permitieron las transmisiones al continente norteamericano.

Si bien, este derecho puede ser sometido a limitaciones o restricciones, pudiendo entrar en conflicto con otros derechos o intereses que resulten relevantes. Y frente a los mismos el TJUE ha tenido ocasión de manifestarse en relación al derecho a la libertad de expresión, en la sentencia de 6 de noviembre de 2003, Caso Bodil Lindqvist c. Göta hovrätt (Suecia), en la que la demandante había creado una página web con la finalidad de que los feligreses de su parroquia obtuviesen información sobre la confirmación, y en la que hacía menciones de manera humorística la situación de sus compañeros, así como cuestiones sobre su salud., sin haber informado de manera previa sobre ello. Tras su condena en instancias inferiores a una multa, la demandante recurrió expresando sus dudas sobre la posibilidad de una restricción contraria al principio de libertad de expresión. El TJUE entendió que para ponderar los distintos derechos e intereses existen disposiciones en la Directiva 95/46/CE que deben tenerse en cuenta junto a las disposiciones nacionales. Así de esta forma, para poder alcanzar un justo equilibrio entre los mismos se deben buscar en el ámbito nacional las normas adaptadas para su aplicación al caso concreto, puesto que las disposiciones de la Directiva 95/46/CE son muy generales y corresponden a un número diverso de situaciones distintas ${ }^{26}$.

${ }^{26}$ Vid. PIÑAR MAÑAS, J.L. "El derecho a la protección de datos de carácter personal en la jurisprudencia del Tribunal de Justicia de las Comunidades Europeas", Cuadernos de Derecho Público, 19, 2003, págs. 45-90. 
El fundamento de la protección de datos, por lo tanto, protege la dignidad de la persona humana y constituye un ámbito de libertad del individuo, teniendo una concreción inexorable en los derechos sobre la personalidad, concretamente el derecho al honor, la intimidad y la propiedad imagen.

Además, la UE exige la promulgación de leyes estatutarias que regulen el uso de datos. La regla básica implica que el procesamiento de datos personales requiere una base legal y si no hubiera una justificación adecuada en la ley sería una violación de los derechos legales. También, los derechos fundamentales del individuo deben protegerse incluso en ausencia de datos confidenciales o daños al individuo. En este sentido dos decisiones del TJUE sobre la privacidad señalan esta misma cuestión, por una parte, la sentencia de 13 de mayo de 2014 de Google España, y conocida por la introducción del "derecho al olvido", y la sentencia de 6 de octubre de 2015 Schrems aquella que hundió el Safe Harbor. En el caso Google España, el TJUE observó que el procesamiento de datos personales representaba una amenaza inherente a los derechos del sujeto de los datos y, debido a este riesgo, solo se puede llevar a cabo si la ley lo permite y determina la forma en que se utilizará la información. Mientras que en el caso Schrems, el TJUE declaró de forma similar que, para establecer la existencia de una injerencia en el derecho fundamental al respeto de la vida privada, sin importar si la información en cuestión es sensible o si las personas afectadas han sufrido alguna consecuencia adversa a causa de esa interferencia.

\section{II.B.3. El Reglamento (CE) no 45/2001 del Parlamento Europeo y del Consejo}

Con el objetivo de continuar avanzando en la regulación de un sector tan cambiante como es el tecnológico, nuevos elementos normativos afloraron como el caso del Reglamento (CE) n $\mathrm{n}^{\circ} 45 / 2001 \mathrm{del}$ Parlamento Europeo y de Consejo, de 18 de diciembre de 2000, relativo a la protección de las personas físicas en lo que respecta al tratamiento de datos personales por las instituciones y los organismos comunitarios y a la libre circulación de estos datos. Contribuyendo a una mayor precisión de la materia y con el objetivo principal de garantizar una aplicación coherente y homogénea de las libertades fundamentales de las personas en lo relativo al tratamiento de datos, al igual que ofrecer una protección que garantice y especifique las 
obligaciones de los responsables de tratamiento en las instituciones correspondientes ${ }^{27}$.

Incluye las mismas definiciones que la Directiva 95/46/CE, así como el ámbito de aplicación y los principios. Asimismo, contempla en su artículo 10 las categorías especiales de tratamiento, aquellos datos sensibles que precisan una especial protección, prohibiendo tratar aquellos datos personales que revelen el origen racial o étnico, las opiniones políticas, las convicciones religiosas o filosóficas, la pertenencia a sindicatos, y los datos relativos a salud y a la sexualidad. Si bien, también existen excepciones, de tal forma que no se aplicaría si el interesado dio su consentimiento explícito, salvo que las normas internas establezcan que la prohibición no puede quedar sin efecto; cuando el tratamiento sea necesario para cumplir las obligaciones y derechos específicos del responsable del tratamiento en materia de Derecho laboral; cuando el tratamiento sea necesario para salvaguardar los intereses esenciales del interesado o de otra persona, cuando el interesado está física o jurídicamente incapacitado para dar el consentimiento.

No obstante, lo más llamativo del Reglamento es, por una parte, la creación de la figura del Supervisor Europeo para la Protección de Datos de carácter personal, definida como la autoridad de control independiente encargada de controlar el tratamiento de datos personales por parte de las instituciones y organismos comunitarios. Tanto el Supervisor como el Supervisor adjuntos son elegidos para un mandato renovable de 5 años. Los artículos 41, 46 y 47 determinen las funciones y competencias de esta figura pudiendo resumirse en supervisión, consulta y cooperación.

Mientras que, por otro lado, el otro de los grandes puntos fuertes que caracterizó a este Reglamento fue el catálogo de derechos que incluyó el derecho de información al interesado, el derecho de acceso a la información sobre sus datos, el derecho de rectificación, el derecho de bloqueo y de supresión, la notificación a terceros y el derecho de oposición.

27 Según lo recogido tanto en el Considerando 5, como en el Considerando 12, que especifica también el fin de garantizar el respecto efectivo de las normas de protección de los derechos y libertades fundamentales como la libre circulación de los datos entre los Estados, instituciones y organismos de la UE 
II.B.4. La Directiva 2002/58/CE del Parlamento Europeo y del Consejo, de 12 de julio de 2002

Esta Directiva, la cual ya no se encuentra en vigor, trajo consigo una serie de normas relativas al tratamiento de datos personales y a la protección de la intimidad en el sector de las comunicaciones electrónicas. Su objetivo principal fue eliminar el envío de correos electrónicos masivo, o conocido comúnmente como "spam". Se establecieron para ello, una serie de reglas que garantizasen la seguridad y confidencialidad de las comunicaciones en redes de internet y servicios móviles de la UE. El interés de esta norma radica en la regulación de la confidencialidad de las comunicaciones, por un lado, que estableció que los Estados miembros tenían que garantizar mediante su legislación dicha confidencialidad de las comunicaciones y los datos de tráfico. Se prohibían la escucha, la grabación, el almacenamiento u otros tipos de intervención o vigilancia de las comunicaciones y de los datos de tráfico asociados a ellas por personas distintas de los usuarios, sin el consentimiento de los mismos, salvo que se encuentren autorizadas legalmente para hacerlo.

II.B.5. Directiva 2006/24/CE del Parlamento Europeo y del Consejo, de 15 de marzo de 2006

La Directiva 2006/24/CE del Parlamento Europeo y del Consejo, de 15 de marzo de 2006 sobre la conservación de datos generados o tratados en relación con la prestación de servicios de comunicaciones electrónicas de acceso público o de redes públicas de comunicaciones y por la que se modifica la Directiva 2002/58/CE fue aprobada como respuesta a los problemas existentes en la seguridad tras los atentados terroristas perpetrados en Madrid el 11-M en el año 2004 y en Londres el 7-J en $2005^{28}$. Y suponía una excepción a la Directiva 95/46/CE y a la Directiva 2002/58/CE, ya que era una singularidad de la Directiva general y presentaba también una aplicación excepcional $^{29}$. En la misma se obligaba a los proveedores de telecomunicaciones a conservar los datos de tráfico y localización, y todos aquellos que fuesen necesarios para identificar al abonado o usuario, para

${ }^{28}$ Como se desprende de la Declaración sobre la lucha contra el terrorismo, adoptada por el Consejo Europeo el 25 de marzo de 2004, y contemplado en el Considerando 8 de la Directiva, y que surgió a raíz de que se le encargase al Consejo el examen de las medidas para establecer normas sobre la conservación de datos de tráfico de las comunicaciones por parte de los prestadores de servicios.

${ }^{29} \mathrm{Vid}$. ORDÓÑEZ SOLIS, D., La protección judicial de los derechos en internet en la jurisprudencia europea, Reus, Madrid, 2014.

(C) UNED. Revista de Derecho UNED, núm. 25, 2019 
que las autoridades nacionales competentes, en casos específicos pudiesen tener acceso a los mismos. Mientras que los datos necesarios para identificar el destino de una comunicación, continua el artículo, se presentarían respecto a la telefonía el servicio móvil utilizado y en Internet, el servicio empleado en dicho ámbito. También se determinan los datos necesarios para identificar el equipo de comunicación de los usuarios que incluían desde los números de teléfono de origen y destino, hasta la identidad internacional del equipo móvil, el conocido IMEI ${ }^{30}$.

Si bien la STJUE de 8 de abril de 2014, asuntos acumulados C-293/12 y C-594/12, Digital Rights Ireland Ltd y Minister for Communications, Marine and Natural Resources, Minister for Justice, Equality and Law Reform, Commissioner of the Garda Síochána/ Kärntner Landesregierung, Michael Seitlinger, Christof Tschohl y otros, consideró que dicha Directiva constituía una injerencia de gran magnitud y de especial gravedad en los derechos fundamentales relativos a la privacidad y a la protección de datos, lo que provocó su anulación. Esta sentencia respondía a las cuestiones prejudiciales presentadas por parte de Irlanda y Austria que tenían por objeto la validez de la Directiva 2006/24/CE. El TJUE señaló que, por una parte, los datos que deben conservarse permiten saber con qué persona y de qué modo ha podido comunicarse un usuario o abonado registrado, determinar el momento de la comunicación y el lugar desde el cual se produjo, así como conocer la frecuencia de las comunicaciones del usuario registrado con determinadas personas en un periodo concreto. Los cuales, si son considerados como un todo, pueden dar indicaciones precisas sobre la vida privada de dichas personas, en concreto: los hábitos de vida cotidiana, los lugares de residencia ya sean permanentes o temporales, los desplazamientos que pudieran producirse, actividades, así como relaciones sociales y medios sociales frecuentados.

En definitiva, la Directiva 2006/24/CE fue declarada inválida porque infringe el principio de proporcionalidad recogido en el artículo 52.1 de la Carta de los Derechos Fundamentales de la Unión Europea, ya que no regulaba con precisión y rigor las condiciones que debían adoptar los operadores de telecomunicaciones para la conservación de datos sin riesgos. Así como la utilización posterior de dichos datos. No siendo tampoco viable el silencio del legislador comunitario frente a aspectos tan trascendentes, dejando a los Estados

${ }^{30}$ El término IMEI significa International Mobile Equipment Identity, y es un identificador único que tiene cada teléfono móvil, funciona como un carnet de identidad para cada dispositivo. 
miembros que salvasen dicha omisión. Esta sentencia ilustró la fragilidad e inestabilidad de las medidas que chocaban contra el derecho de la protección de datos, y la necesidad de cerrar la brecha entre la UE y sus ciudadanos pudiendo superar de esta forma el déficit de confianza en este ámbito ${ }^{31}$.

II.B.6. El Reglamento (UE) no 2016/679 del Parlamento Europeo y del Consejo de 27 de abril de 2016

Como último elemento configurativo normativo se encuentra el Reglamento (UE) 2016/679 del Parlamento Europeo y del Consejo de 27 de abril de 2016 relativo a la protección de las personas físicas en lo que respecta al tratamiento de datos personales y a la libre circulación de estos datos y por el que se deroga la Directiva 95/46/CE, el conocido RGPD. El RGPD se ha ido desarrollando desde 2009, debido a la rápida evolución tecnológica y la necesidad de uniformidad del régimen jurídico en materia de protección de datos, publicando la Comisión Europea una propuesta oficial a principios de $2012{ }^{32}$. No obstante, no ha sido hasta 2018, la entrada en vigor del mismo. Con este cambio se pretende mejorar el nivel de protección y armonización de datos personales en toda la UE, puesto que la anterior Directiva ya no cumple con los requisitos de privacidad del entorno digital actual ${ }^{33}$. La legislación sobre privacidad de datos, como se ha ido mencionando, ha ido evolucionando con el desarrollo de tecnologías de recopilación y procesamiento de datos personales. De tal manera que este nuevo reglamento tiene como objetivo cumplir con los desafíos actuales relativos a protección de datos personales, a fortalecer los derechos de privacidad en línea y a impulsar la economía digital de la UE.

El primer capítulo del RGPD abarca las disposiciones generales, incluyendo la indicación de sujetos obligados, objeto, ámbito material y territorial, así como la definición de los elementos principales. El RGPD en su artículo cuarto desarrolla una serie de términos, muchos de los cuales ya estaban definidos en la anterior Directiva

${ }^{31} \mathrm{Vid}$. BALLASCHK, J., "In the unseen realm: transnational intelligence sharing in the European Union- Challenges to fundamental rights and democratic legitimacy", Stanford Journal of International Law, 51, 2015, págs. 19-51.

32 Vid. CARDENAS ARTOLA, I., Protección de datos (memento experto), Lefebvre - El Derecho, Madrid, 2012.

${ }^{33}$ Vid. DE HERT, P., y PAPAKONSTANTINOU, V., "The new general data protection regulation: still a sound system for the protection of individuals?", Computer Law \& Security Review, 32, 2016, págs. 179-194. 
95/46/CE. De esta forma, en el caso de datos personales se mantiene la concepción preexistente incluyendo solamente los datos de localización y los identificadores en línea como información, resultando interesante la mención en el Considerando 27 a la no aplicación a los datos de personas fallecidas, dejando la posibilidad de que los Estados establezcan normas específicas sobre ello. Se añaden diferentes definiciones con el fin de actualizar la normativa. Junto a los nuevos conceptos y definiciones dadas el legislador europeo optó por afianzar también una serie de principios jurídicos que, aunque tuvieron su comienzo en la Directiva 46/95/CE y en la normativa desarrollada en cada país, la jurisprudencia del TJUE ha ido expandiendo y desarrollando. Como novedad que incluyó el RGPD, se encuentra la limitación del tratamiento de los datos personales a la prestación del consentimiento expreso, o a las disposiciones legales, ya no tiene cabida el consentimiento tácito, como venía ocurriendo por ejemplo en el caso español. Si bien, cuando no produzca alguna de las situaciones anteriores, no tiene por qué suponer necesariamente la ilicitud del tratamiento, siempre y cuando, se realice una ponderación sobre la prevalencia del interés legítimo. Se han visto ampliados los derechos de los interesados y ha aparecido dos nuevas figuras en este campo, el delegado de protección de datos, como supervisor del cumplimiento en materia de protección de datos, y las evaluaciones de impacto, para comprobar qué riesgos pueden entrañar los nuevos tratamientos de datos por parte de una entidad. La reforma que ha propuesto el RGPD está basada en un modelo de cumplimiento, que presenta una gestión enfocada al riesgo.

\section{LA VISIÓN NORTEAMERICANA DEL CONCEPTO PRIVACY Y LA PROTECCIÓN DE DATOS}

Una vez analizado el derecho de protección de datos en el ámbito europeo y su desarrollo normativo resulta necesario entrar a conocer la visión norteamericana. Sin embargo, a modo introductorio, y para entender el sistema americano sobre la protección de datos y las transferencias de datos, así como los acuerdos existentes con la UE sobre esto, se hace necesaria una breve contextualización del sistema jurídico imperante en EE.UU. Como es sabido, hablamos de una república federal constitucional y una democracia representativa que ha heredado de los británicos la tradición jurídica del common law en su mayoría, aunque actualmente se puede hablar de un sistema jurídico combinado, pues no es un sistema puro de precedentes ni consiste exclusivamente en la codificación. Por lo tanto, 
en el derecho estadounidense las decisiones judiciales tienen fuerza de ley en la jurisdicción que corresponda, proviniendo esta fuerza de los conceptos de precedente y stare decisis. Hablamos de precedente judicial cuando una decisión de un tribunal constituye una autoridad obligatoria para el mismo tribunal y para otros de igual o inferior rango, mientras que el principio de stare decisis impone una dinámica que implica que los jueces han de decidir los casos del presente atendiendo a cómo se decidieron con anterioridad casos parecidos, y forzando la vinculación del juez a sus decisiones anteriores. Este principio es la razón del establecimiento de los precedents al igual que una fuente de legitimidad de las decisiones de los tribunales $^{34}$. Por lo tanto, la tradición del common law otorga margen de libertad a los jueces para crear leyes en áreas importantes, como por ejemplo sobre cuestiones sobre marcas y patentes, así como en los contratos.

En lo referente a la legislación americana, en un primer lugar se encontraría la Constitución federal de los EE. UU, como ley suprema y central en el derecho y en la política norteamericana. Mientras que, en el ámbito federal se encuentra la Constitución federal y las propias leyes federales, incluyendo reglamentos ejecutivos de este ámbito, recopiladas en el United States Code (U.S.C.) que es una compilación de la legislación federal general que se enfoca sobre cuestiones donde existe una expresa cesión de competencia sobre el gobierno federal a través de la Constitución, como es la defensa nacional, la política monetaria, o la propiedad intelectual o industrial, entre otros. En un tercer lugar, se encontraría la legislación estatal, ya que los cincuenta estados americanos cuentan con soberanías independientes con sus propias constituciones estatales, reteniendo poderes para promulgar leyes relativas a materias que no se encuentren expresamente supeditas a la competencia federal, y codificándose la mayor parte de su normativa en muchos casos a través de códigos de leyes. Esta legislación incluiría el derecho constitucional estatal, la legislación del estado particular y los reglamentos de los condados y ciudades conocidos como ordenanzas. Sin embargo, como la Constitución estadounidense limita a las áreas determinadas la capacidad del Gobierno federal para legislar, y como delega la autoridad para legislar solamente en el Congreso, no existe un com-

${ }^{34} \mathrm{Vid}$. AHUMADA RUÍZ, M.A., "Stare decisis y creación judicial de derecho (Constitucional). A propósito del precedente constitucional en el sistema judicial norteamericano, de Ana Laura Magaloni Kerpel”, Revista Española de Derecho Constitucional, 67, 2003, págs. 351-365. 
mon law federal genérico que podría ser equivalente a lo establecido en los estados ${ }^{35}$.

Si bien, la evolución que se ha producido de forma independiente de las leyes en la mayor parte de los estados dio lugar a grandes divergencias entre las leyes de los diferentes estados. Por lo que, para entender de forma completa el Derecho americano resulta imprescindible examinar la jurisprudencia con el fin de determinar de qué manera los jueces han ido interpretando los diferentes problemas jurídicos en torno al ámbito de la privacidad y la protección de datos, y por qué los acuerdos adoptados con la UE han adoptado esa forma y ese contenido.

\section{III.A. El reconocimiento de la privacidad}

La primera y principal diferencia existente en este sistema es que no existe un derecho constitucional análogo o similar al derecho a la protección de datos como el de la UE. A pesar de que el right to be alone fue reconocido inicialmente entre los tribunales americanos a través de Warren y Brandeis y su artículo publicado en 1890 "The Right to Privacy", como consecuencia de la expansión del periodismo sensacionalista basado en escándalos y rumores sobre la vida de personas famosas. Ambos juristas argumentaron que el common law podía desarrollar una protección de la privacidad, sobre la capacidad de los individuos de comunicar y extender sus pensamientos, sentimientos y emociones hacia otros. Entendiendo que este derecho no provenía de la propiedad si no que procedían del genérico derecho a estar solo. No obstante, no fue hasta 1902 con el caso Roberson c. Rochester Folding Box $\mathrm{Co}^{36}$, cuando se produjo un verdadero reconocimiento de la privacidad. En este caso Franklin Mills utilizó una litografía de Abigail Roberson para la realización de un anuncio sin su consentimiento, generando en la demandante un daño a su honor y su nombre por las consecuencias derivadas de la publicación del anuncio. Sin embargo, la Corte no reconoció la acción porque no había precedentes sobre casos similares y que sería necesaria la aparición de nueva legislación sobre esta temática. Esta argumentación generó gran debate entre las diferentes escuelas de derecho americanas, provocando oleadas de críticas ante la pasividad de la Corte. Esto provocó que un año más tarde se promulgase un esta-

35 Vid. FLETCHER, G.P., y SHEPPARD. S., American Law in A Global Context. The Basics, Oxford University Press, Nueva York, 2005.

${ }^{36}$ Roberson v. Rochester Folding Box Co., 64 N.E. 442 (1902). 
tuto que estableciese una causa de acción para los casos de invasión de la privacidad. Y, en 1905 la Corte Suprema de Georgia reconoció la invasión de la privacidad como un acto ilícito civili7 a través del caso Pavesich v. New England Life Insurance Co. ${ }^{38}$, donde también había sido utilizada la imagen del demandante sin consentimiento.

Unas décadas más tarde en 1960, uno de los grandes juristas expertos en tort law, William Prosser, reconoció cuatro ilícitos diferentes sobre la privacidad en los casos surgidos a raíz del artículo de Warren y Brandeis ${ }^{39}$ :

- La intrusión en la intimidad, implica que aquellas personas que intencionalmente se inmiscuyan en los asuntos privados, están sujetos a responsabilidad por la invasión de la privacidad de ese tercero, si dicha intrusión resultase altamente ofensiva.

- La revelación pública de datos privados, supone que aquellos que den publicidad a un asunto relacionado con la vida privada de otra persona, se encontrará sujeta a responsabilidad por invasión a la privacidad, si dicho asunto resulta altamente ofensivo y no resulta una preocupación que legítimamente puedan conocer las personas del círculo privado de dicha persona.

- "False light", este caso es similar a la difamación, ya que las leyes de privacidad en los EE. UU incluyen el derecho que tiene una persona, que no forma parte de la vida pública, a la protección frente a la publicidad que puedan hacer sobre ella que resulte poco favorecedora y no deseada. La diferencia entre la difamación y el false light, es que la primera aborda la reputación y la segunda está vinculada a la dignidad.

- Apropiación, que supone que quien se apropia de para su propio uso o beneficio del nombre o de la semejanza del nombre de otra persona, está sujeta a la responsabilidad derivada de la invasión de su privacidad.

Los cuales son reconocidos actualmente en la mayoría de los estados americanos, siendo uno de los últimos en reconocerlos el es-

37 Se reconoció un common law tort for privacy invasions, siendo los torts los actos ilícitos civiles de carácter extracontractual para los que se prevé una reparación. Esta rama del derecho en el sistema estadounidense que regula los derechos y obligaciones derivados de los actos ilícitos se conoce como tort law.

38 Pavesich v. New England Life Insurance Co., 50 S.E. 68 (1905).

39 Vid. PROSSER, W.L., "Privacy”, California Law Review, 48, 1960, págs. 383423.

(C) UNED. Revista de Derecho UNED, núm. 25, 2019 
tado de Minnesota en Lake v. Wal-Mart Stores Inc ${ }^{40}$. Si bien, junto a dichos ilícitos surgió paralelamente el incumplimiento de confidencialidad, en un primer momento en el ámbito médico y referido a la confidencialidad que debe mantenerse sobre los datos de salud entre el médico y su paciente. El caso Simonsen v. Swenson $(1920)^{41}$ reconoció que la relación entre doctor y paciente requería una férrea confidencialidad ya que en numerosas ocasiones el paciente debía proporcionarle datos sobre su vida personal y situaciones que en otras circunstancias no estaría dispuesto a compartirlos. No obstante, se ha ido reconociendo este incumplimiento en aquellos casos en los que se produce una brecha en el deber de lealtad y hay un beneficio como consecuencia del mismo ${ }^{42}$.

Por su parte, también se entendió que la privacidad do la intimidad debía ser entendida como un retiro voluntario y temporal de una persona de la sociedad, lo que no debe suponer una ausencia de información sobre la misma, sino que, más bien, que ésta cuenta con una plena capacidad de control sobre la información existente y que pueda concernirle y, por tanto, permitir o denegar el acceso a otras personas ${ }^{43}$. No obstante, hubo una escasa acogida de este planteamiento, como se comprobará más adelante por causa de la relevancia otorgada por los tribunales al alcance de la Primera Enmienda sobre la libertad de prensa y lo medios de comunicación como los guardianes del poder. Por ello, la intimidad se sigue concibiendo como un derecho de libertad frente a las intromisiones del poder público ${ }^{44}$.

\section{III.B. La tendencia legislativa norteamericana sobre la privacidad}

Como se adelantaba anteriormente no existe un derecho constitucional reconocido como tal sobre la protección de datos en el ámbito norteamericano, además su Constitución tampoco obliga al gobierno a tomar acciones encaminadas a crear condiciones que permitan la existencia de derechos fundamentales. Y en el caso del sector público solamente hay un interés limitado en lo referente a la privacidad de la información que protege a las personas cuando

${ }^{40}$ Lake V. Wal-Mart Stores, Inc., 582 N.W.2.d 231, 235 (1998).

${ }^{41}$ Simosen V. Swenson, 177 N.W 831 (1920).

${ }^{42}$ Vid. SOLOVE, D.J., y SCHWARTZ. P.M., Information Privacy Law, Aspen Publishers, Nueva York, 2015.

${ }^{43}$ Vid. WESTIN, A.F., Privacy and freedom, Nueva York, Atheneum, 1970.

${ }^{44}$ CARRILLO, M., El derecho a...op. cit., p. 120y ss. 
el gobierno procesa la misma. De esta forma, en el ámbito constitucional, la jurisprudencia ha vinculado ese interés implícito en la libertad de asociación garantizada en la Primera Enmienda ${ }^{45}$, salvaguardando frente a cualquier obligación estatal la revelación de permanencia a un grupo u organización; en la Cuarta Enmienda ${ }^{46}$, que protege a los individuos contra diversos tipos de recabo de información personal por parte del gobierno. Sin embargo, este texto queda obsoleto frente a las condiciones de los usos gubernamentales de los datos personales y las bases de datos actuales. Ya que realmente solamente proporciona una garantía o protección frente a registros y detenciones arbitrarías. Así, por ejemplo, la Constitución no protege a una persona en aquellos casos en los que un tercero, como su entidad bancaria, entrega su información personal al gobierno. Además, los circuitos federales han desarrollado jurisprudencia que establece que el derecho constitucional sobre la privacidad de la información será protegido con el uso estatal cuando éste se realice sin un mandato legal expreso, una política pública determinada o por interés público reconocible.

Ante esta situación el escrutinio constitucional por parte de los tribunales frente a esta cuestión tiende a ser poco exigente ${ }^{47}$. Por ello y comparado con la UE, EE. UU carece de cualquier derecho análogo a la protección de datos, y es que las salvaguardias constitucionales más importantes en el ámbito de la información personal en EE. UU se refieren al libre flujo de datos, y no a la privacidad personal. Desde el punto de vista del sector público, existe un interés limitado sobre la protección de la privacidad sobre información de las personas, restringiéndose a los casos donde el gobierno es el que procesa los datos personales.

A diferencia de la legislación europea, en la legislación norteamericana existe un principio de flujo de información libre que permite el procesamiento de cualquier información personal a menos que una ley limite dicha acción. Además, la legislación de privacidad de

${ }^{45}$ La Primera Enmienda se recoge del siguiente modo: "El Congreso no hará ley alguna con respecto a la adopción de una religión o prohibiendo la libertad de cultos; o que restrinja la libertad de expresión o de prensa, o el derecho del pueblo a reunirse pacíficamente y a solicitar al gobierno la reparación de agravio”.

${ }^{46}$ La traducción a la Cuarta Enmienda sería la siguiente: "El derecho del pueblo a la seguridad en sus personas, domicilios, papeles y efectos, contra registros y detenciones arbitrarias, será inviolable, y no deben exigirse garantías, sino por una causa probable, apoyada en un Juramento o afirmación y describiendo particularmente el lugar que deba ser registrado y la persona o las cosas que deben confiscarse".

${ }^{47}$ Vid. SCHWARTZ, P.M., y PEIFER, K.N., "Transatlantic Data Privacy Law", The Georgetown Law Journal, 106, 2017, págs. 121-178. 
la información incluye estatutos y reglamentos a nivel federal y estatal, no existe una ley general. Y es que en contraste con la concepción de la UE la protección de datos se presenta como un derecho fundamental, mientras que en EE. UU la privacidad de la información queda relegada al ámbito mercantil, con el objetivo de proteger a los consumidores y promover la competencia y evitar actos o prácticas engañosas en el comercio. Existe una clara orientación de las leyes hacia el ámbito de mercado, intentando favorecer a los consumidores sobre su privacidad, sobre todo el ámbito de intercambio de datos y en relaciones tales como la obtención de crédito, las transacciones financieras o el visionado de vídeos. También se extienden a los datos de salud, en atención a aquellos pacientes que reciben atención médica de entidades que llevan a cabo transmisiones electrónicas de información a seguros o fines similares.

Se ha buscado la protección de las compañías tecnológicas y de su crecimiento activo por los beneficios que presentaban en el mercado americano, de ahí que la normativa sobre la privacidad sea más laxa que en el ámbito europeo.

\section{CONCLUSIONES}

A lo largo de la exposición anterior se ha podido comprobar que la protección de las personas físicas en relación con el tratamiento de datos personales es un derecho reconocido en el ámbito de la UE. Desde la aparición del concepto jurídico de privacidad y los diversos avances tecnológicos la UE ha considerado que los datos personales tienen un estatus jurídico alto, y ha buscado armonizar los enfoques de cada una de las naciones europeas para poder generar una normativa destinada a proteger la privacidad. Esta línea de pensamiento se ha traducido en las diferentes regulaciones surgidas a lo largo de los años, afectando sobre todo al ámbito de las transferencias internacionales de datos, puesto que no todos los países fuera de la UE tienen la misma consideración sobre la protección de datos, como ocurre en el caso de EE. UU y su escasa normativa al respecto. En la UE el interés por parte de los responsables de tratamiento de los datos está sujetos a una prueba de proporcionalidad, a limitaciones y sanciones cuando infringen los derechos en el ámbito de protección de datos. En EE. UU, en contraposición, la protección normativa más fuerte no recubre a los particulares cuyos datos son tratados, si no para los propios responsables de tratamiento. Como se ha explicado anteriormente los Estados Unidos carecen de un derecho equivalente al de protección de datos europeo. Además, tam- 
poco presentan un mandato constitucional que requiera que los responsables de tratamiento tengan una base legal para el uso de datos personales. De ahí la necesidad de llevar a cabo acuerdos o sistemas que garanticen o ayuden a prevenir problemas jurídicos entorno a estos terceros países, alcanzando de esta forma un punto de conciliación.

La UE ha construido una normativa en torno a los derechos de la identidad digital de sus ciudadanos, promoviendo una cultura con fuertes protecciones constitucionales en los diferentes países que la componen y leyes generales que restringen el alcance de las entidades que buscan el tratamiento de datos personales de los ciudadanos. Por su parte, en EE. UU la privacidad carece de una protección constitucional similar y presenta un enfoque legislativo sectorial, ya que cuentan con un mercado interesado en el libre flujo de datos.

No obstante, tanto la UE como EE. UU reconoce el valor de la privacidad y del flujo de información y de datos personales, de tal forma que, a pesar de las diferencias entre ambos el sistema de mercado a nivel global obliga a una interacción constante entre estos gigantes. La cuestión será si dichos sistemas pueden salvar las diferencias sobre los elementos fundamentes que componen la privacidad y la protección de datos en cada una de las culturas legales. Por ello, documentos normativos como el RGPD y el Privacy Shield ofrecen nuevas oportunidades para la resolución de conflictos por las concepciones diferentes sobre la privacidad.

\section{BIBLIOGRAFÍA}

Aiello, J. y Aiello, T.C. (1974). "The development of personal space: proxemics behavior of children six through sixteen", Human ecology, 2, 127-136.

Ahumada Ruíz, M.A., "Stare decisis y creación judicial de derecho (Constitucional). A propósito del precedente constitucional en el sistema judicial norteamericano, de Ana Laura Magaloni Kerpel”, Revista Española de Derecho Constitucional, 67, 2003, págs. 351365.

BALLASCHK, J., "In the unseen realm: transnational intelligence sharing in the European Union- Challenges to fundamental rights and democratic legitimacy", Stanford Journal of International Law, 51, 2015, págs. 19-51. 
BRANDEIS, L.D., y WARREN, S.D., "The right of privacy (the implicit made explicit)", Harvard Law Review, 5, 1890, págs.193-220.

Cardenas Artola, I., Protección de datos (memento experto), Lefebvre - El Derecho, Madrid, 2012.

CARRILlo, M., El derecho a no ser molestado. Información y vida privada, Aranzadi, Navarra, 2003.

Davara Rodríguez, M., Manual de Derecho Informático, Thomson Reuters, Pamplona, 2015.

De Hert, P., y Papakonstantinou, V., "The new general data protection regulation: still a sound system for the protection of individuals?”, Computer Law \& Security Review, 32, 2016, págs. 179194.

Fletcher, G.P., y ShePPARD. S., American Law in A Global Context. The Basics, Oxford University Press, Nueva York, 2005.

HERRÁN ORTIZ, A.I., El derecho a la protección de datos personales en la sociedad de la información, Universidad de Deusto, Bilbao, 2003.

KUnER, C., European data privacy law and online business, Oxford University Press, Nueva York, 2003.

LóPez Chao, V.A., El impacto del diseño del espacio y otras variables socio-físicas en el proceso de enseñanza-aprendizaje, Tesis doctoral Universidad de la Coruña, 2016.

MAestre Rodríguez, J.A., "La intimidad: El derecho de autodeterminación personal", en C. VILLAGRASA ALCAIDE (coord.), Nuevas tecnologías de la información y derechos humanos, (págs. 275-284), Cedecs, Barcelona, 2003.

MARTínez DE Pisón, J. "Vida privada e intimidad: implicaciones y perversiones”, Anuario de Filosofía del Derecho, 14, 1997, págs. 717738.

MaRTínez DE Pisón, J., "El derecho a la intimidad: de la configuración inicial a los últimos desarrollos en la jurisprudencia constitucional”, Anuario de Filosofía del Derecho, 32, 2016, págs. 409-430.

Medina Guerrero, M., La protección constitucional de la intimidad frente a los medios de comunicación, Tirant lo Blanch, Valencia, 2005.

ORDÓÑEz Solis, D., La protección judicial de los derechos en internet en la jurisprudencia europea, Reus, Madrid, 2014. 
Ortí Vallejo, A., Derecho a la intimidad e informática, Tutela de la persona por el uso de ficheros y tratamientos informáticos de datos personales. Particular atención a los ficheros de titularidad privada, Comares, Granada, 1994.

Pérez Luño, A.E., Derechos humanos, Estado de Derecho y Constitución, Tecnos, Madrid, 1986.

Perry, A., Rubinsten, O., Peled, L., y Shamay-Tsoory, S., "Don't stand so close to me: A behavioral and ERP study of preferred interpersonal distance", Neuroimage, 83, 2013, págs-761-769.

Prosser, W.L., "Privacy”, California Law Review, 48, 1960, págs. 383423.

PiÑAR MAÑAS, J.L. "El derecho a la protección de datos de carácter personal en la jurisprudencia del Tribunal de Justicia de las Comunidades Europeas", Cuadernos de Derecho Público, 19, 2003, págs. 45-90.

Rebollo Delgado, L., (Vida privada y protección de datos en la Unión Europea, Dynkinson, S.L, Madrid, 2008.

REDING, V., "The upcoming data protection reform for the European Union”, International Data Privacy Law, 1, 2011, págs. 3-5.

Ruíz Miguel, C., La configuración constitucional del derecho a la intimidad, Tecnos, Madrid, 1995.

Savirimuthu, J., Security and Privacy. Volume III, Routledge, Oxford, 2016.

Solove, D.J., y Schwartz. P.M., Information Privacy Law, Aspen Publishers, Nueva York, 2015.

Schwartz, P.M., y PeIfer, K.N., "Transatlantic Data Privacy Law”, The Georgetown Law Journal, 106, 2017, págs. 121-178.

TEJERInA Rodríguez, O., Seguridad del estado y privacidad, Reus, S.A, Madrid, 2014.

Tinnefeld, M.T., Buchner, B., Petri, T., y Hof, H.J., Einführung in das datenschutzrecht. Datenschutz und informationsfreiheit in Europäischer sicht, De Gruyter Oldenbourg, Berlin, 2012.

Westin, A.F., Privacy and freedom, Nueva York, Atheneum, 1970. 